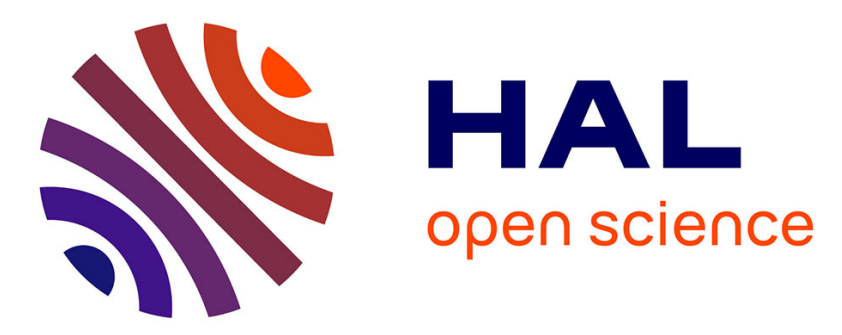

\title{
La palissade dans tous ses états : l'enclos du Britzgyberg (Illfurth, Haut-Rhin) et autres aménagements palissadés dans les habitats du premier âge du Fer
}

\author{
Anne-Marie Adam
}

\section{- To cite this version:}

Anne-Marie Adam. La palissade dans tous ses états: l'enclos du Britzgyberg (Illfurth, Haut-Rhin) et autres aménagements palissadés dans les habitats du premier âge du Fer. Archimède: archéologie et histoire ancienne, 2016, 3, pp.49-59. 10.47245/archimede.0003.ds1.06 . hal-01587232

\section{HAL Id: hal-01587232 \\ https://hal.science/hal-01587232}

Submitted on 13 Sep 2017

HAL is a multi-disciplinary open access archive for the deposit and dissemination of scientific research documents, whether they are published or not. The documents may come from teaching and research institutions in France or abroad, or from public or private research centers.
L'archive ouverte pluridisciplinaire HAL, est destinée au dépôt et à la diffusion de documents scientifiques de niveau recherche, publiés ou non, émanant des établissements d'enseignement et de recherche français ou étrangers, des laboratoires publics ou privés. 


\section{DOSSIER THÉMATIQUE : DES FOSSÉS ET DES REMPARTS. ENCEINTES ET SITES FORTIFIÉS DU RHIN SUPÉRIEUR ENTRE PROTOHISTOIRE ET MOYEN ÂGE}

\section{Olivier BUCHSENSCHUTZ}

Avant-propos. Des enceintes en terre anhistoriques à Google Earth

8 Lizzie SCHOLTUS

Histoire de la recherche dans le bassin de Saint-Dié-des-Vosges

20 Maxime WALTER

Les sites de hauteur du massif vosgien. Actualisation des données et modalités d'implantation

37 Jean-Jacques SCHWIEN

Chateaux et enceintes des Vosges du Nord. Topographie et longue durée

49 Anne-Marie ADAM

La palissade dans tous ses états : I'enclos du Britzgyberg (Illfurth, Haut-Rhin) et autres aménagements palissadés dans les habitats du premier âge du Fer

60 Clément FÉLIU

L'enceinte inférieure du Frankenbourg (67) et les remparts à poteaux frontaux de la fin de l'âge du Fer dans l'espace du Rhin supérieur. Pour une révision de la typologie des Pfostenschlitzmauern

74 Jacky $\mathrm{KOCH}$ et Thomas FISCHBACH

Enceintes de hauteur en pierres et formes « primitives » de châteaux ? L'exemple du Bernstein

87 Adrien VUILLEMIN

Les enceintes urbaines en moyenne Alsace (1200-1850)

102 Jean-François PININGRE

Les enceintes de l'âge du Bronze et du premier âge du Fer en Franche-Comté. Un bilan des recherches

124 Clément FÉLIU et Jean-Jacques SCHWIEN

Conclusion. Nouvelles perspectives sur les enceintes du Rhin supérieur

\section{ACTUALITÉ DE LA RECHERCHE : ARCHÉOLOGIE DES RÉSEAUX}

\section{Claire CAMBERLEIN}

Les réseaux en archéologie : approche historiographique et interdisciplinaire

135 Thomas HUTIN

Lieux d'échanges et espaces publics en Gaule à La Tène finale

150 Steeve GENTNER

Économie du fer et voies de communication, de l'abattage du minerai à la distribution du métal : I'exemple du nord de la Forêt-Noire au Ve siècle av. J.-C.

169 Loup BERNARD et Rémy WASSONG

Du Danemark au Fossé rhénan. Un siècle d'analyse des voies de communications protohistoriques : évolution des méthodes et mise en commun des données

184 Steeve GENTNER et Rémy WASSONG

Conclusion. L'archéologie des réseaux : une thématique aux multiples facettes

\section{VARIA}

187 Fábio VERGARA CERQUEIRA

To march in phalanx, to jump with weights, to tread the grapes, to knead the bread. What is the aulos for?

206 Hermann AMON Les supra-commandements comme solution à la crise militaire du III siècle de l'Empire romain sous Philippe l'Arabe et Gallien

218 Martina BONO

Il processo di Cremuzio Cordo in Dio LVII, 24, 2-4

\section{LA CHRONIQUE D'ARCHIMÈDE}

228 Frédéric COLIN (éd.)

La Chronique d'Archimède. Bilan des activités scientifiques 2015-2016 de I'unité mixte de recherche 7044 


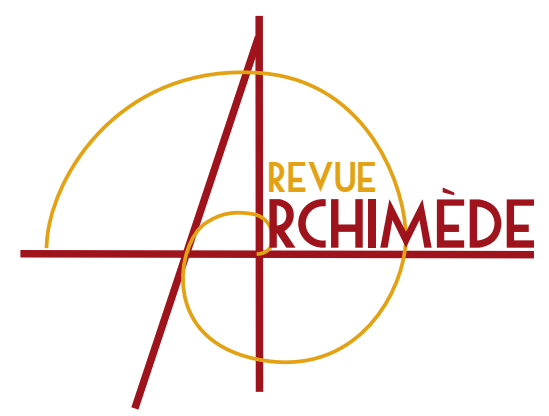

\section{LA PALISSADE DANS TOUS SES ÉTATS : L'ENCLOS DU BRITZGYBERG (ILLFURTH, HAUT-RHIN) ET AUTRES AMÉNAGEMENTS PALISSADÉS DANS LES HABITATS DU PREMIER ÂGE DU FER}

Anne-Marie ADAM

Professeure émérite Université de Strasbourg UMR 7044 Archimède

anne.marie.adam@unistra.fr

\section{RÉSUMÉ}

Les fouilles du site de hauteur hallstattien du Britzgyberg (Illfurth, dép. Haut-Rhin) ont permis la mise au jour et I'étude d'un imposant système palissadé qui, durant une partie du Hallstatt D1, a dû jouer un rôle important dans la structuration du site. A partir du rapprochement de ce dispositif avec des palissades monumentales, présentes dans d'autres habitats privilégiés du premier âge du Fer en Europe moyenne, on peut s'interroger sur les fonctions de ces architectures : aménagements à valeur
Mots-CLÉs

Période hallstattienne, habitat fortifié,

Herrenhof, architecture de bois, enclos. défensive ou constructions structurant l'espace et matérialisant le prestige et le pouvoir d'un notable?
The excavations in the Britzgyberg hillfort (Illfurth, dép. Haut-Rhin) allowed to discover and study a strong palisade, which must have played a keypart in the settlement's organisation during part of the Hallsatt D1. We can wonder about the role of this constructions, through the comparisons with monumental palisades excavated in other major settlements in Europe during the first Iron Age : are they defence installations or structuring elements whichmaterialize the prestige and the power of a dignitary?
KeYwords

Hallstatt period, fortifiedsettlements, Herrenhof, wooden architecture, 


\section{LA GRANDE PALISSADE DU BRITZGYBERG ET SES AMÉNAGEMENTS ANNEXES}

Conduites depuis une dizaine d'années, les fouilles du site de hauteur hallstattien du Britzgyberg, sur la commune d'Illfurth (France, Haut-Rhin), ont permis la mise au jour et l'étude d'un imposant système palissadé qui, durant une partie du Hallstatt D1 (dans le courant de la $1^{\text {ère }}$ moitié du $\mathrm{VI}^{\mathrm{e}}$ siècle avant notre ère) paraît jouer un rôle important

Figure 1 : plan général du site avec emplacement des zones de fouille (état 2014) et développement de la grande palissade (DAO S. Fichtl).

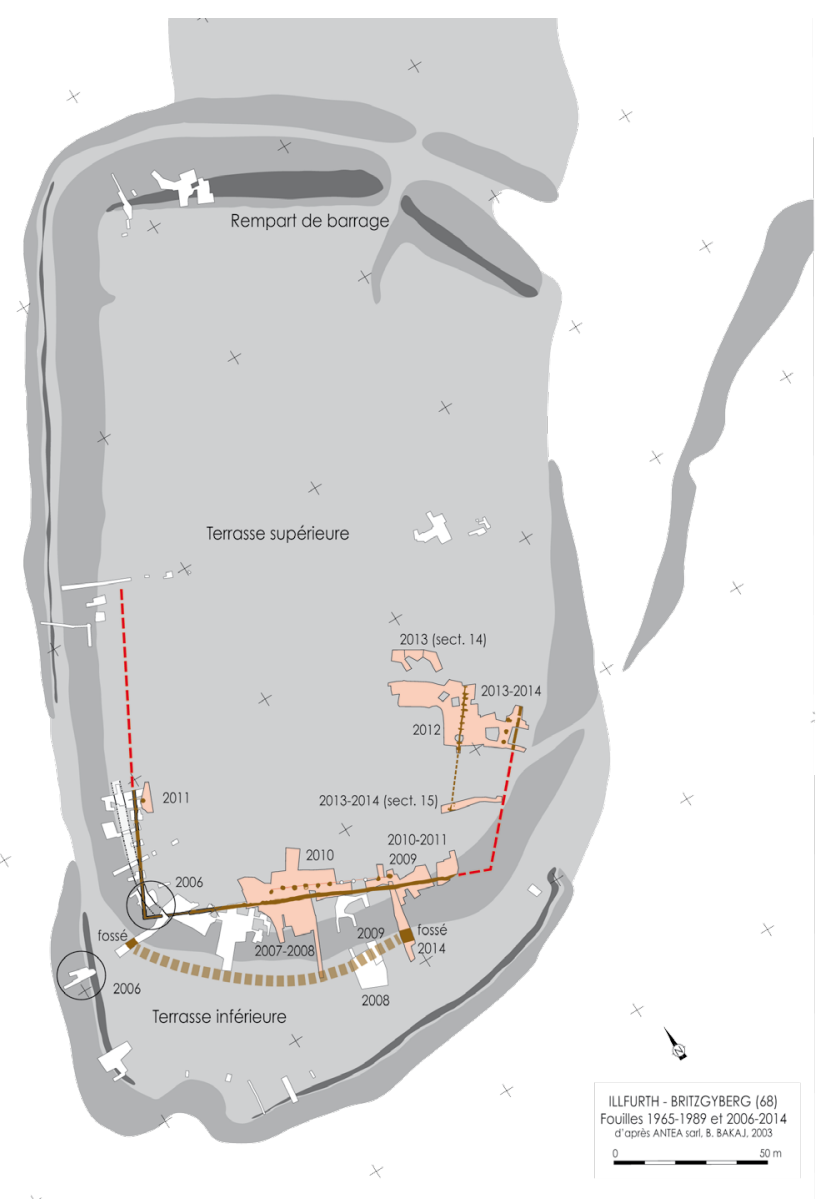

dans la structuration du site [1] (fig. 1). Ce dispositif est, sur trois côtés au moins du plateau, matérialisé par une grande tranchée rectiligne, large en moyenne de 0,80 m pour une profondeur conservée - à partir du niveau du substrat - qui oscille entre $1,55 \mathrm{~m}$ et $1,90 \mathrm{~m}$. Sur de courts tronçons, toutefois, on a identifié du côté sud, des portions où les dimensions de la tranchée deviennent soudain beaucoup plus modestes, sans qu'on comprenne encore les raisons de ce changement (fig. 2). Traversant toute la largeur du plateau sur ce flanc méridional de la butte (soit environ $100 \mathrm{~m}$ de développement), la tranchée a été également suivie, côté ouest, sur un tronçon continu de $44 \mathrm{~m}$ de long, perpendiculaire au précédent. Lors d'une récente campagne, menée en août-septembre 2015, sur la bordure nord-ouest du plateau, la même tranchée a pu être identifiée de nouveau sur ce flanc occidental, de même qu'elle avait pu être fouillée sur quelques mètres, en 2013-2014, longeant la bordure orientale (voir plan du site, fig. 1). Dans la plupart de ces zones de fouilles, le passage d'une palissade continue (constituée de poteaux jointifs d'une vingtaine de centimètres de section) est bien visible dans les coupes et sur le fond de la tranchée (sous la forme de traces de charbon de bois ou à partir de la position des pierres de calage) (fig. 3). La palissade est parfois installée au centre de la tranchée, mais le plus souvent décentrée vers l'un des bords. Le matériau calcaire extrait lors du creusement de la tranchée constitue l'autre partie, bien reconnaissable, du comblement.

Cette construction, mise en évidence par les fouilles récentes sur trois côtés, était probablement complétée par un quatrième côté, comme on peut le déduire des indications fournies par les précédents fouilleurs du site, Roger et Joël Schweitzer. Ces derniers ont en effet, dans les années 1980, réalisé plusieurs coupes et sondages dans le talus qui délimite le site protohistorique du côté nord, le plaçant, au moins à partir d'un certain moment de son

[1] AdAm 2010a, p. 368-371. 


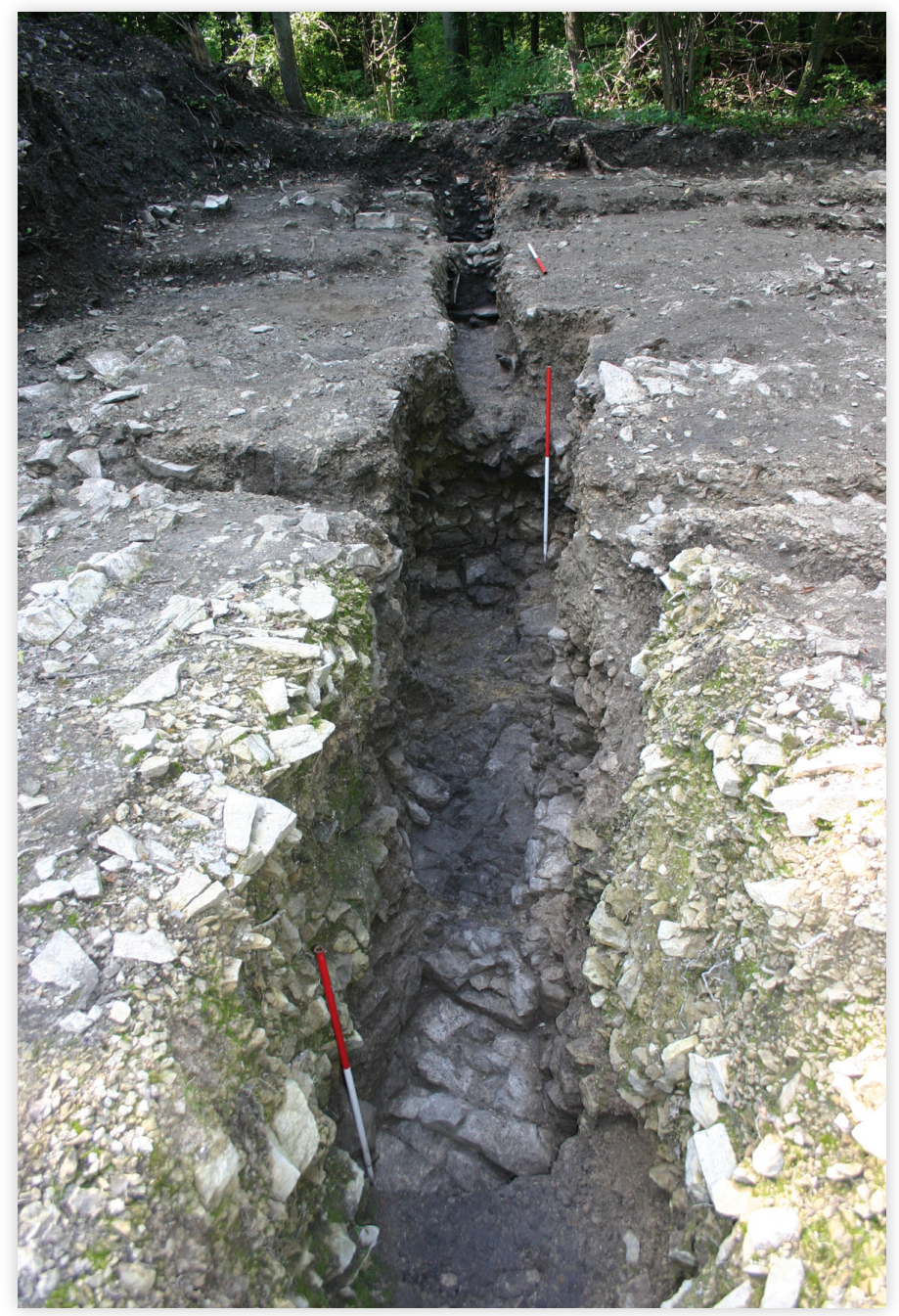

Figure 2 : vue d'ensemble de la grande tranchée de palissade (tronçon sud-2008) ; au fond, rétrécissement de la structure (photo A.-M. Adam).

histoire, dans la catégorie des éperons barrés. Même si la stratigraphie présentée par ces fouilleurs pour le secteur du rempart de barrage doit être envisagée avec circonspection, la phase la plus ancienne y est décrite comme une grande tranchée de palissade, analogue à celle qu'ils avaient déjà fouillée à l'angle sud-ouest [2]. Après destruction de la palissade, cette tranchée est scellée par un niveau (interprété par R. et J. Schweitzer comme un niveau d'habitat) dont l'homogénéité chronologique n'est pas assurée, mais qui comporte du mobilier du Hallstatt D2/D3 (dont deux fibules à timbale conique sur l'arc) et fournit donc un terminus ante quem pour la datation de la structure la plus ancienne, logiquement attribuable au Hallstatt D1. C'est par-dessus le tout qu'est ensuite édifié le rempart de barrage proprement dit.

La question qui se pose alors est de savoir si nous avons affaire, dans tous les tronçons reconnus, à différents côtés de plusieurs enclos contigus ou voisins occupant le sommet du plateau, ce qui serait plus conforme à l'image fournie par d'autres sites, mieux connus, qui peuvent nous offrir des comparaisons (comme La Heuneburg, ou le Mont-Lassois,

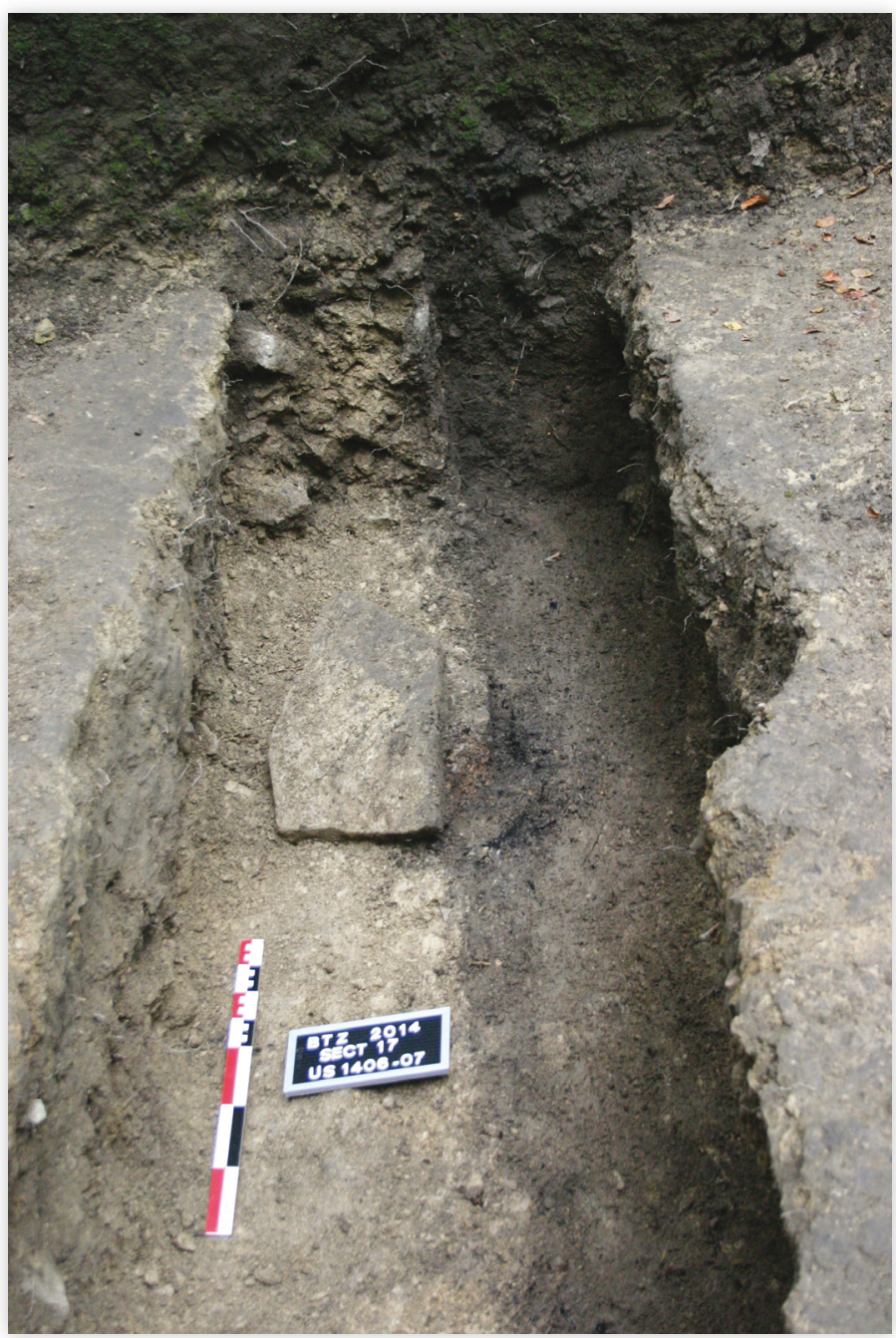

Figure 3 : grande tranchée de palissade, tronçon est (2014): trace du passage de la palissade, comblement calcaire et pierre de calage (photo A.-M. Adam).

infra), ou s'il s'agit d'un dispositif unique, long de $235 \mathrm{~m}$ environ, c'est-à-dire d'un vaste enclos couvrant près de trois hectares. Dans cette seconde hypothèse, aucun parallèle précis ne peut être mentionné, du moins compte tenu de la situation perchée de cet ensemble. En effet, si I'on sait maintenant que, dans les sites privilégiés du Premier âge du Fer, l'espace est souvent structuré au moyen de grandes palissades rectilignes, l'image offerte est souvent différente. Sur le plateau sommital du Mont-Lassois (plateau de SaintMarcel), à Vix (France, Côte-d'Or), plusieurs enclos, de superficie variable, et enfermant des constructions de taille et sans doute de fonctions différentes, s'échelonnent le long d'un axe central de circulation. Le schéma qui se dessine ainsi est probablement celui d'une résidence seigneuriale, organisée autour de plusieurs espaces complémentaires (zones résidentielles ou de représentation, zones d'exploitation) [3]. À La Heuneburg, sur le haut Danube (Allemagne,

[2] SCHWEITZER 1997, p. 63.

[3] MilCent 2012, p. 96-101. 


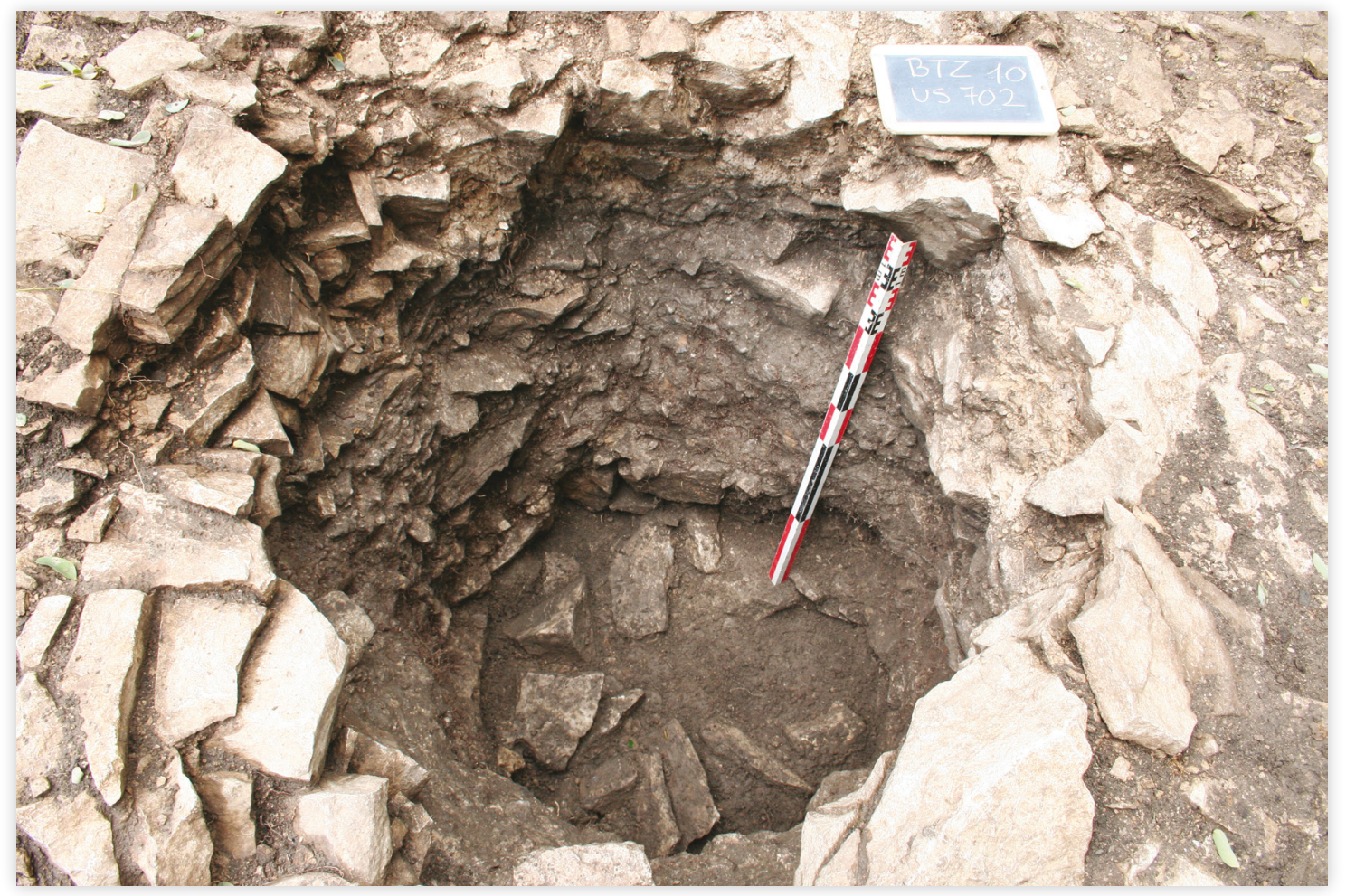

$<$ Figure 4

Trou de poteau appartenant à l'alignement sur le flanc sud (photo A.-M. Adam).

$\checkmark$ Figure 5

Trou de poteau à proximité du tronçon est de la palissade : comblement calcaire induré et fantôme du pilier quadrangulaire (photo A.-M. Adam)

Bade-Wurtemberg), une vaste « agglomération extérieure », dont on peut actuellement estimer la superficie à près de cent hectares, se compose d'unités foncières juxtaposées, sous la forme d'établissements enclos, regroupés en quartiers que délimitent fossés et talus [4]. Un système analogue d'enclos est visible aussi dès le début de son occupation (au début du Hallstatt D1, au tournant du VII ${ }^{\mathrm{e}}$ et $d u v^{\mathrm{e}}$ siècle avant J.-C.) sur la butte qui constitue le cœur du complexe, et S. Kurz suppose une sorte de topographie hiérarchique, où les seigneurs les plus puissants s'installent sur la butte, tandis que les chefs de clans secondaires se regroupent au pied de celle-ci.

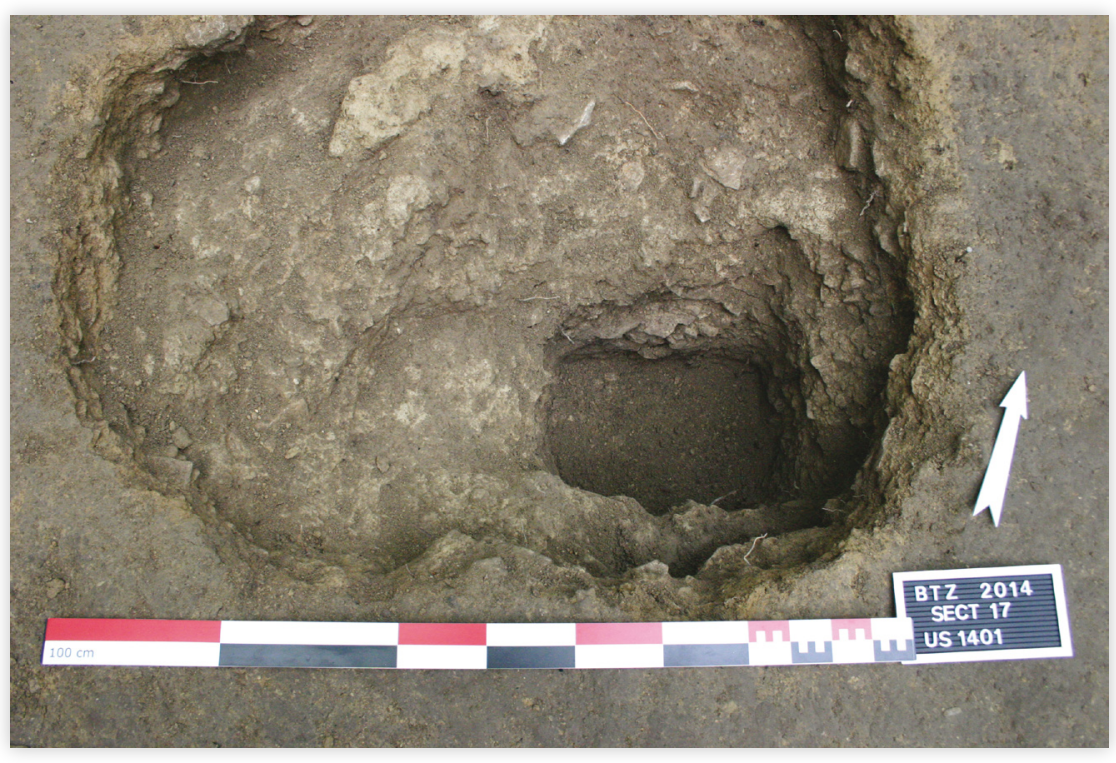

Au Britzgyberg, un autre élément architectural vient compléter ponctuellement le dispositif d'enclos et contribue à lui conférer un caractère exceptionnel, au moins dans l'état actuel de nos connaissances. À $3 \mathrm{~m}$ en retrait de la tranchée, des alignements de trous de poteau ont été, en effet, mis en évidence sur plusieurs tronçons, à chaque fois parallèles à la palissade. Le gabarit de ces creusements est en accord avec celui de la palissade : ils présentent un diamètre moyen de $1,20 \mathrm{~m}$ et la profondeur conservée peut atteindre jusqu'à 1,84 m. L'entraxe, de centre à centre, est plus ou moins régulier $(3,75 \mathrm{~m}$ à $4 \mathrm{~m})$, mais l'ajustement de la ligne a été obtenu de façon empirique et les poteaux une fois mis en place apparaissent souvent décentrés à l'intérieur du trou. Huit de ces structures ont été fouillées côté sud ; une seule pour l'instant côté ouest ; trois autres

repérées, dont une seule intégralement fouillée, sur le flanc est. Côté sud, on peut en restituer onze en tout, formant un alignement continu sur environ $40 \mathrm{~m}$ de long, et plus ou moins centré par rapport à la longueur totale de l'aile sud de la palissade. Plusieurs indices montrent que les « poteaux » ainsi installés étaient en fait des piliers de section quadrangulaire (de 0,30 m sur 0,40 m environ) (fig. 4 et 5). En l'absence, semble-t-il, d'un aménagement continu tout le long de la face interne de l'enclos, il est difficile de reconstituer son aspect et sa fonction. On peut dire, tout au plus, que dans l'état actuel des recherches, I'hypothèse de bâtiments jouxtant la palissade paraît peu

[4] KURz 2010, p. 247-249. 


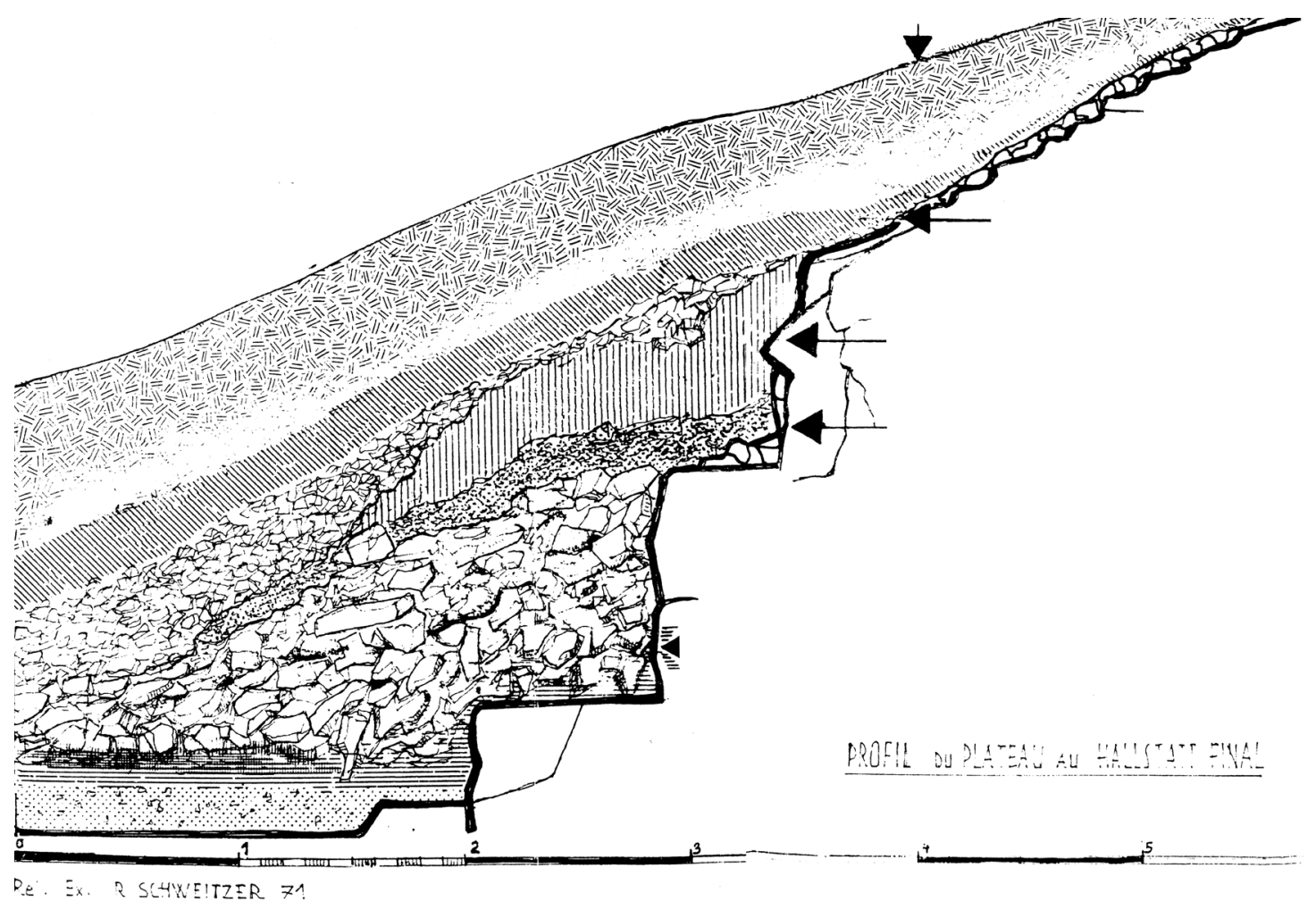

Figure 6

Relevé de coupe réalisé par R. Schweitzer en 1971 à la pointe sud du plateau (coupe S 1971/1).

vraisemblable, et qu'il faut plutôt privilégier I'hypothèse d'une sorte de portique, doublant intérieurement, par endroits, la grande palissade et renforçant son caractère monumental. On peut restituer au-dessus la présence (elle aussi ponctuelle ?) d'un espace de circulation de type « chemin de ronde ». En tout cas, et par opposition à certains remparts que nous évoquerons plus loin, on ne dispose d'aucun indice archéologique pour l'existence d'une accumulation de terre (talus) entre la palissade et la rangée de piliers.

S'il ne dessine pas un rectangle régulier, puisque, compte tenu de l'écart d'orientation entre les branches méridionale et orientale, l'angle sud-est, non localisé exactement à ce jour, doit présenter une ouverture un peu supérieure à $90^{\circ}$, I'enclos ne s'adapte pas non plus par sa forme au relief naturel dont il n'épouse pas les contours. C'est plutôt, à l'inverse, le paysage qui semble avoir été en partie façonné par I'homme, de manière à compléter et mettre en valeur l'ouvrage palissadé. En effet, des travaux de terrassement paraissent avoir été entrepris (sans doute contemporains de la construction de la palissade) pour créer un effet de gradin(s), qui lui-même surmonte un fossé de bonne largeur, encerclant la base de la «terrasse ». L'effet de terrasses aujourd'hui visible dans la topographie du plateau, avec ses deux niveaux emboîtés, ne correspond donc pas à une formation naturelle, mais résulte très probablement d'une intervention anthropique sur le paysage. Une réflexion sur le profil géologique de la butte menée par Dominique Schwarz (Faculté de Géographie, Université de Strasbourg) confirme le caractère artificiel de ce relief. D'une manière générale, ce type de replat n'a que trois origines naturelles possibles (en dehors des situations particulières liées à des coulées volcaniques) : des failles, des replats structuraux, liés à des alternances de couches dures et tendres, ou des dépôts de formations superficielles, de type terrasse alluviale. Ces trois types de formation pouvant être exclus ici pour différentes raisons, il convient donc d'écarter I'hypothèse d'une origine naturelle de ces replats.

À partir de sondages réalisés à plusieurs reprises au travers de la pente de la terrasse supérieure, R. et J. Schweitzer ont pu établir que, si dans la partie supérieure de la pente, le substrat calcaire montre une déclivité naturelle et régulière, ce dénivelé s'accentue brusquement à partir du milieu de la pente, où la roche a visiblement été retaillée par paliers successifs (fig. 6). Ils expliquaient ces gradins par la nécessité d'extraire des matériaux de construction, notamment pour les structures défensives du site, et l'irrégularité relative des entailles d'un secteur à l'autre pourrait paraître confirmer cette explication. Pourtant de nouvelles coupes ouvertes entre 2008 et 2014, à plusieurs dizaines de mètres des sondages précédents, ont révélé la même situation, et notamment la présence de deux marches (dont I'une haute de plus de $1 \mathrm{~m}$ ) taillant artificiellement le substrat, prolongées par un replat de quelques mètres de large, lui aussi aménagé par l'homme, avant que ne s'ouvre le fossé (fig. 7 et 8 ).

La persistance du même dispositif sur près de $100 \mathrm{~m}$ de long, c'est à dire tout autour de la pointe sud du plateau, I'association des gradins et du fossé ne sont pas en accord 


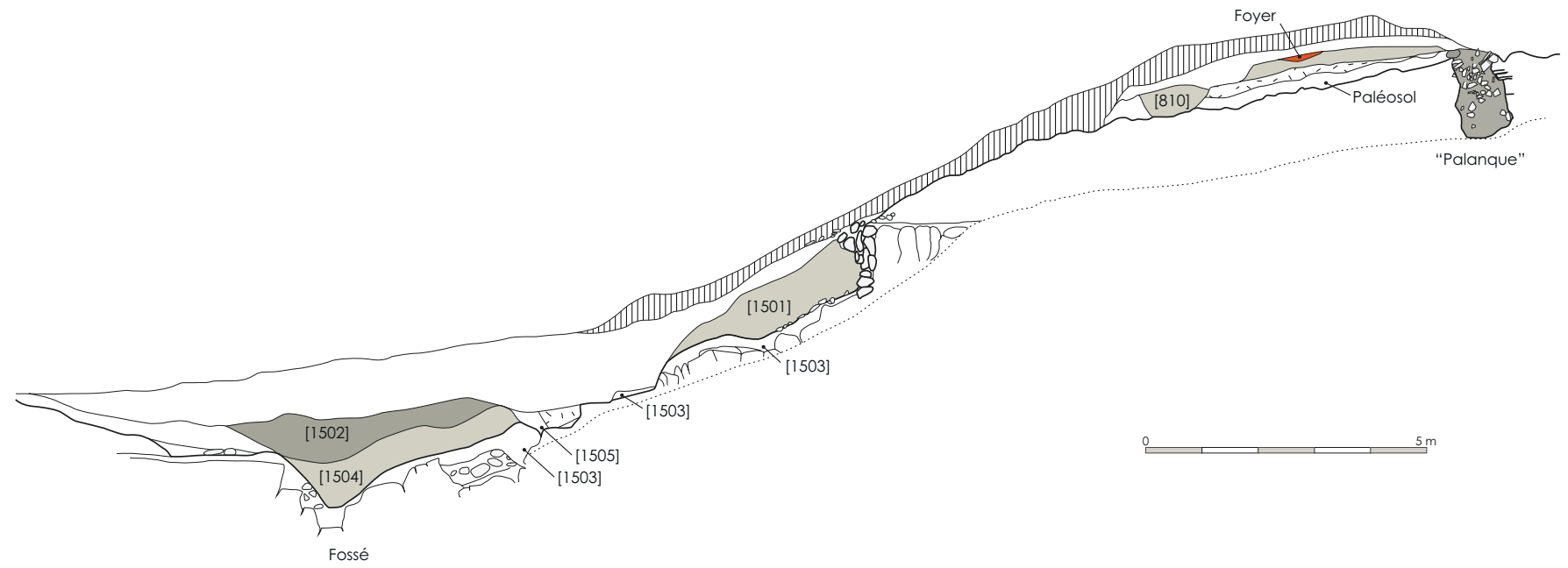

Figure 7 : coupe à travers la pente de la terrasse supérieure et le fossé inférieur (2014) (DAO S. Fichtl).

avec I'hypothèse d'une simple carrière de matériaux de construction, et correspondent plus certainement à un aménagement concerté. Il est probable que celui-ci se prolonge vers l'ouest et vers l'est, et notamment le fossé, mais on ignore encore si ce dernier entoure toute la terrasse. Quelle était la fonction d'un tel fossé ? Même si les dimensions de l'ouvrage sont assez importantes, nous avons noté que ses deux bords présentent une déclivité modeste. Par ailleurs, rien dans la nature de son comblement $n^{\prime}$ indique qu'il ait été durablement empli d'eau. On peut supposer plutôt un fossé sec, laissé ouvert et entretenu, et on doit s'interroger sur son éventuelle valeur défensive. La même question se pose d'ailleurs à propos du (ou des) gradin(s) ménagés dans la pente. La succession des aménagements complique évidemment l'accès par la pente au plateau supérieur, qu'elle contribue dans une certaine mesure à fortifier. Mais I'objectif premier peut être aussi de faire ressortir, de loin, le caractère imposant du dispositif de clôture constitué par la grande palissade (fig. 8).
Il est intéressant de noter qu'on trouve de la même façon à La Heuneburg, autre site «princier » dans le sud de I'Allemagne, un fossé de contour, cernant le pied de la butte sur plusieurs dizaines de mètres au moins, à la base de son flanc nord-ouest. Ce fossé, qui devait présenter à l'origine une profondeur de plus de $6 \mathrm{~m}$, a été mis en place au Hallstatt D1. II n'était pas lié directement au rempart qui cernait, lui, le bord supérieur de la butte, mais renforçait peut-être le système défensif et contribuait surtout à mettre en valeur la pente, déjà raide et peut-être accentuée encore par des travaux de terrassement, ainsi que l'enceinte qui la couronnait. Ce fossé paraît avoir perdu de son importance durant le dernier quart du vi ${ }^{\mathrm{e}}$ siècle avant J.-C., et avoir été assez vite comblé, peut-être dans le cadre d'une profonde réorganisation du système défensif qui a suivi la destruction du rempart de briques crues [5].

[5] Bofinger \& Goldner-Bofinger 2008, p. 218 et 225.

\section{Figure 8}

Hypothèse de restitution de la grande palissade et de son portique interne (avec le fossé de contour et la pente aménagée) (DAO S. Fichtl).

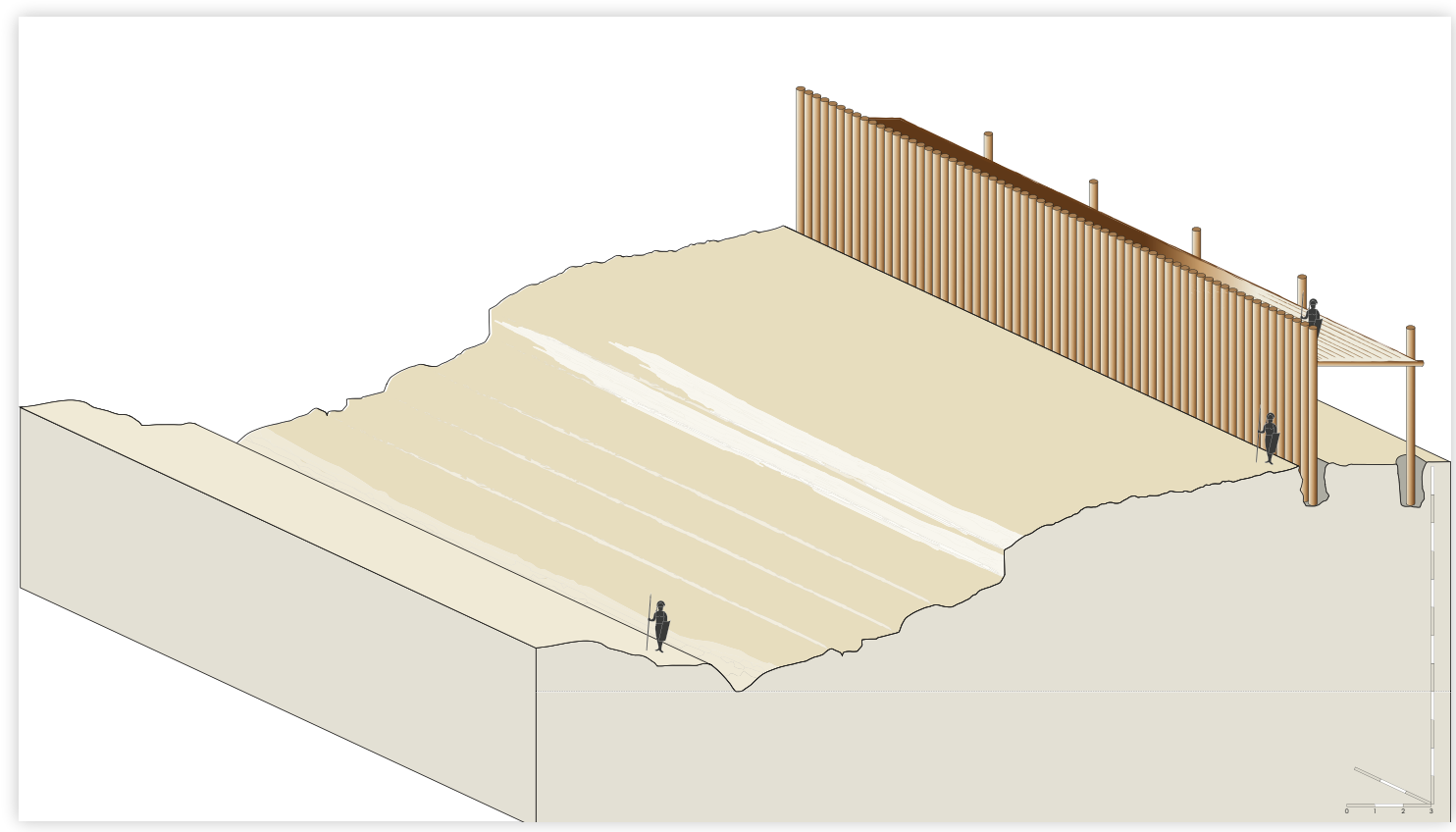


Compte tenu des éléments que nous venons de décrire, et des comparaisons qui s'offrent à nous, se pose donc la question de la fonction de l'ensemble architectural en bois du Britzgyberg, et, dans le cadre d'une réflexion plus large sur les enceintes protohistoriques, celle de sa valeur défensive. Devons-nous considérer cette construction, complétée par la pente aménagée et le fossé inférieur, comme une fortification, ou comme un enclos monumental, pourvu d'un rôle principalement ostentatoire ? Formellement, la grande palissade se distingue de façon radicale des constructions défensives aménagées postérieurement sur la butte : le rempart de barrage nord et le rempart de contour qui vient compléter, sans doute dans la dernière phase d'occupation du site hallstattien, le dispositif des fortifications. Ces constructions qui, au moins pour le rempart de barrage, ont pu connaître plusieurs phases, présentent toutes des caractéristiques architecturales « classiques » pour l'âge du Fer (remparts à poutrage interne et/ou à poteaux verticaux, de type «Pfostenschlitzmauer ») [6]. Tout au plus peut-on noter que le rempart de contour se singularise par la présence, au lieu du plus habituel parement de façade en pierres, d'un front en bois (probablement une palissade à poteaux jointifs), maintenu à l'aval par un massif de pierres [7].

\section{DES REMPARTS PROTOHISTORIQUES EN BOIS ?}

Dans le cas de la grande palissade, plus ancienne, le matériau utilisé ne constitue pas, en lui-même, un critère discriminant pour la distinction entre « enclos » et « enceinte », et le fait qu'on se trouve, au Hallstatt D1, face à une construction réalisée entièrement en bois ne suffit pas pour exclure totalement que l'édifice ait pu jouer un rôle défensif. En dehors des remparts à front palissadé, comme le rempart de contour du Britzgyberg évoqué plus haut, des palissades simples peuvent prendre place dans l'évolution architecturale de certains ouvrages fortifiés, dont elles ne constituent pas nécessairement l'état le plus « primitif ». C'est plus largement, semble-t-il, dans les remparts du Bronze final que le bois prend une place importante, comme on le voit, par exemple, dans l'enceinte du Bullenheimer Berg (Allemagne, Bavière) [8], dans la construction de laquelle le bois peut être décliné de diverses façons selon les phases. Mais le plus souvent il n'est pas employé seul : I'ouvrage peut se présenter comme une simple palissade formée de rondins horizontaux maintenus par des pièces

[6] Schweitzer 1997, p. 59-63; Adam 2010b.

[7] AdAM 2010b, p. 39-42.

[8] DieMER 1991, p. 26-30, fig. 4-7.
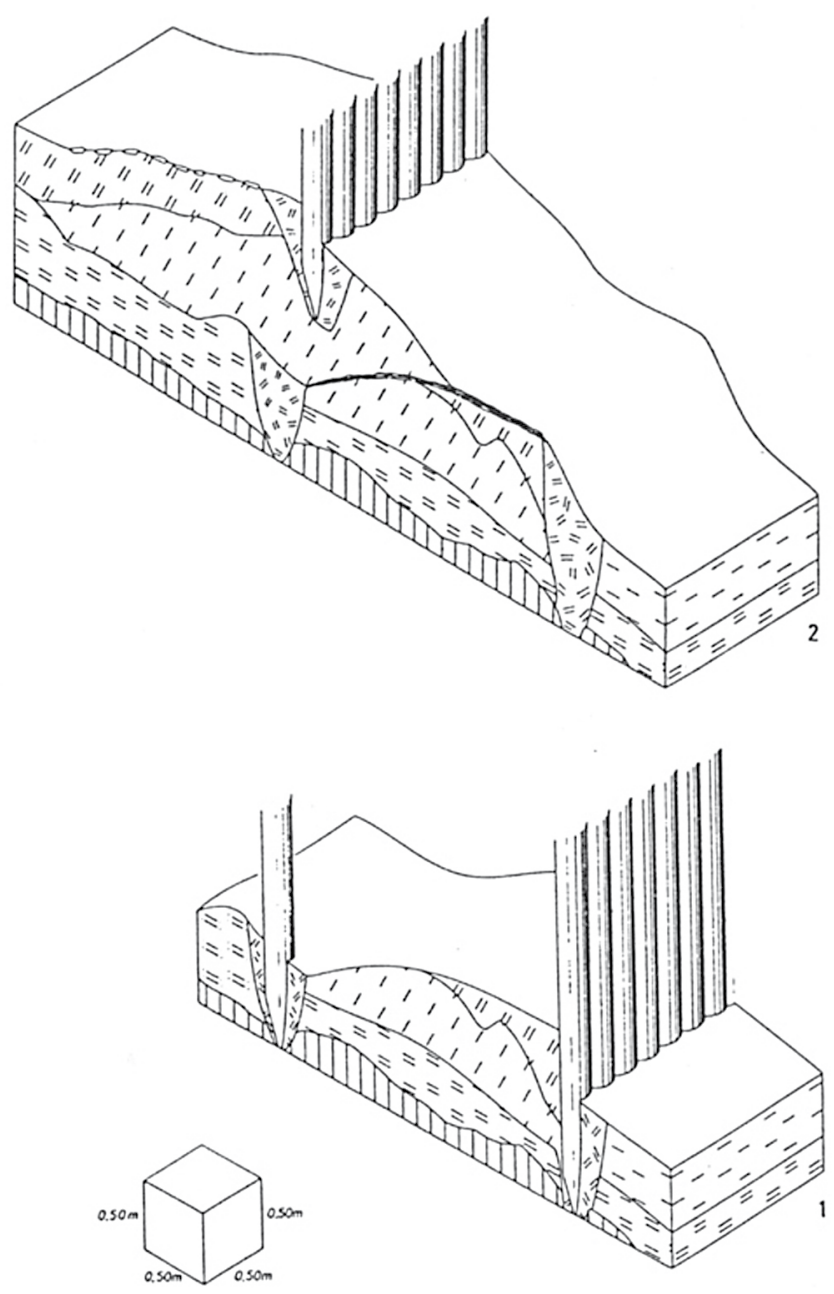

Figure 9 : schémas des deux états du rempart protohistorique du site fortifié de Lübbecke « Babilonie » (d'après BÉRENGER 1999, fig. 8).

verticales, mais son pied est taluté ; à d'autres époques, il s'agit d'une construction en bois plus complexe, adossée à un massif de terre dans lequel elle est ancrée.

La comparaison entre notre enclos et le rempart protohistorique du site fortifié de Lübbecke «Babilonie » (Allemagne, Westphalie) permet de mettre en évidence à la fois les ressemblances et les différences entre ces deux types d'ouvrages. Sur ce site de hauteur, plusieurs lignes de fortification ont été repérées, dont l'une, qui rentre dans la catégorie des enceintes de contour, appartient à l'époque protohistorique. Cette fortification, où le bois joue un rôle important, témoigne elle-même de deux états de construction (fig. 9) : dans une première phase, une palissade frontale est associée à un talus, sur lequel elle s'appuie ; à l'arrière, à $3 \mathrm{~m}$ environ, un poteau isolé est associé au même état, sans que, à travers la simple coupe réalisée, son rôle puisse être précisé. Dans le second état, une nouvelle palissade est installée en retrait par rapport à la précédente, couronnant une nouvelle accumulation de matériaux. Entre ces deux phases, sans doute 
assez rapprochées dans le temps, une couche d'incendie, apparemment contemporaine de la destruction du premier état, a permis d'obtenir une datation C 14 correspondant à La Tène ancienne.

On peut considérer que cette comparaison nous aide à résoudre au moins une question de terminologie, en matérialisant clairement la différence entre le « rempart » et «l'enclos », en particulier à travers la présence, dans le premier cas, d'une accumulation de matériaux (talus) associés à la construction de bois. Mais, si elle autorise à préciser le vocabulaire utilisé, elle ne permet pas de résoudre la question d'une éventuelle fonction défensive des enclos.

\section{AUTRES ENCLOS PALISSADÉS DU PREMIER ÂGE DU FER}

En dehors du plateau du Britzgyberg, on peut citer d'autres cas de sites dans lesquels des palissades, d'aspect plus ou moins monumental, jouent un rôle important dans la délimitation ou dans la structuration de l'établissement. La «Göllersreuther Platte », près de Landersdorf (Allemagne, Bavière, Lkr. Roth), dans le Jura franconien, est un site fortifié d'un peu moins d'un hectare de superficie, cerné d'un rempart de contour, avec une porte côté sud, dont une aile est conservée. À l'intérieur de cette fortification, la pointe occidentale du plateau est isolée par un dispositif de clôture dont les fondations sont creusées dans le rocher, et qui enferme une surface d'environ $2600 \mathrm{~m}^{2}$. Les fouilles réalisées par Markus Schußmann ont permis de reconnaître les différents éléments associés dans ce dispositif [9]. Une petite tranchée de palissade d'environ $40 \mathrm{~cm}$ de profondeur, au fond de laquelle se lisent des traces de poteaux, est doublée à $7 \mathrm{~m}$ à l'est, et donc côté

\section{Figure 10}

Proposition de restitution de la clôture intérieure du site de Landersdorf« Göllersreuther » (d'après SCHUSSMANN 2012, fig. 32).

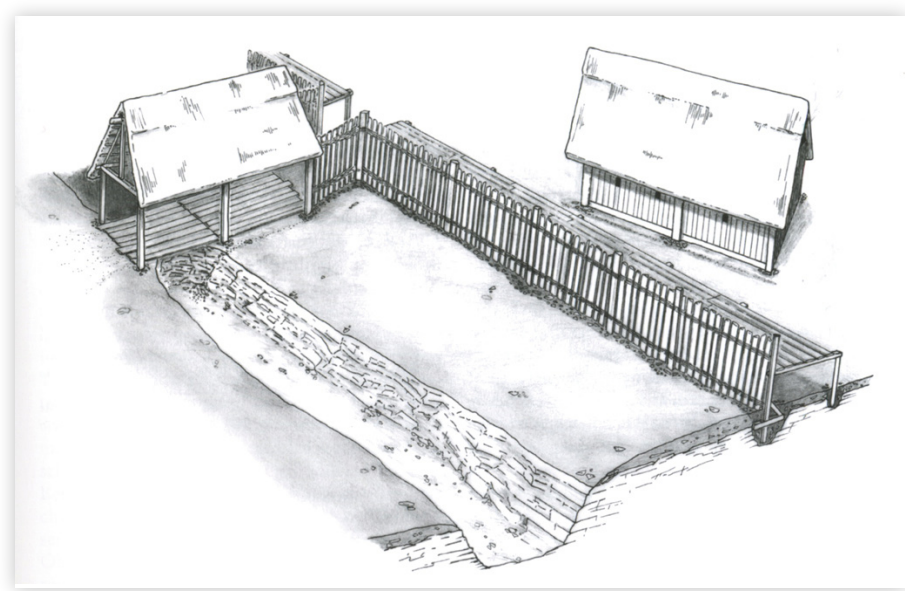

externe, par un fossé large de 2à 2,5 m et profond de 55 à $60 \mathrm{~cm}$, qui lui est parallèle. Vers l'intérieur (à une distance de 1,20 à 1,50 m à partir de la palissade), une ligne de trous de poteau a été interprétée par le fouilleur comme les vestiges de l'aménagement d'un chemin de ronde. L'entrée dans cet enclos « réservé » était aménagée, des trous de poteau signalant l'existence probable d'un porche, plus ou moins imposant, associé à un retour de la palissade et à une passerelle traversant le fossé (fig. 10). Comment faut-il interpréter ces constructions ? Doit-on privilégier I'aspect militaire et en faire une sorte de redoute fortifiée ? Ou bien s'agit-il d'une résidence « seigneuriale », isolée du reste de l'occupation par un dispositif architectural qui en matérialise le statut?

Ce cas d'un enclos qui paraît réservé, isolé du reste des constructions, dans un angle de l'habitat, se rencontre probablement sur le site du Goldberg, dans le Nördlinger Ries (Allemagne, Bade-Wurtemberg) [10]. On y accède par un porche monumental, peut-être une tour-porche, et une nouvelle subdivision interne abrite deux bâtiments, de bonne taille et reposant sur des poteaux de fort diamètre, ce qui peut laisser supposer que ces édifices possédaient également une certaine hauteur. Dans ce dernier cas, la monumentalité d'une architecture de prestige paraît l'emporter sur les considérations défensives. On a donc proposé $d^{\prime} y$ voir un complexe résidentiel et de représentation pour le chef de la communauté [11].

L'établissement de Oberhaching « Kyberg » (à proximité de Munich) [12] est implanté sur l'extrémité d'un promontoire, délimitée par les deux ailes d'un système palissadé, qui isolent une superficie d'environ $4000 \mathrm{~m}^{2}$. Le promontoire lui-même a été modifié dans sa topographie : en effet le remblaiement d'un léger thalweg qui l'entaillait côté est a permis un élargissement de sa surface. Ces travaux ont précédé la seconde phase d'aménagements sur le site, au moment où I'habitat, précédemment ouvert, s'est trouvé enclos par l'installation d'une palissade, interrompue au niveau d'une entrée, que délimitaient deux petits fossés perpendiculaires. Un fossé externe a peut-être déjà été creusé à cette époque, afin (selon l'hypothèse proposée par les fouilleurs du site) de renforcer cette enceinte palissadée plutôt légère et « fragilisée » par une large interruption au niveau de l'entrée. On remarque que I'interprétation ici avancée pour ce système de clôture intègre clairement la

[9] Schussmann 2012, p. 65-69.

[10] PARZINGER 1998, p. 76-78.

[11] On connaît moins bien, en revanche, la situation et les aménagements internes des enclos palissadés repérés sur certains sites de hauteur de la fin de l'âge du Bronze, comme au Frauenberg, qui domine le monastère de Weltenburg, sur les bords du Danube : Schauer 2000-2001, p. 77, fig. 3-4.

[12] Pätzold \& Schwarz 1961. 
dimension défensive, sur un site que sa situation topographique apparente, de fait, à un « éperon barré ».

Dans un second état de la palissade, la porte est rétrécie et forme maintenant un corridor de $10 \mathrm{~m}$ de long, de même profondeur probablement que les ailes de la porte initiale, mais plus étroit que celle-ci. On installe aussi une seconde palissade en retrait par rapport à la précédente. Dans un troisième temps, est creusé un fossé interne, qui s'interrompt symétriquement de part et d'autre de l'entrée. Comme ce fossé frôle dans l'angle sud-ouest la palissade précédente, on peut supposer que celle-ci n'était plus en service, et a été remplacée par un troisième tracé, situé plus en retrait encore. Cet habitat n'ayant jamais été publié intégralement, on ne dispose pas d'éléments de chronologie qui puissent permettre d'évaluer le laps de temps qui s'écoule entre ces différents états ; I'ensemble du site couvre le Hallstatt $D\left(\mathrm{VI}^{\mathrm{e}}\right.$ siècle avant J.-C.). L'évolution du système de clôture a donc pu être assez rapide (fig. 11) : faut-il en conclure qu'on a cherché à plusieurs reprises à en améliorer les propriétés défensives ? Ou que la multiplication des lignes imbriquées les unes dans les autres, au cours de la dernière phase, a plutôt une valeur symbolique et ostentatoire?

\section{Figure 11}

Évolution du système de délimitation de l'établissement de Oberhaching « Kyberg » (d'après PÄTzOld \& SchWARz 1961, pl. 2).

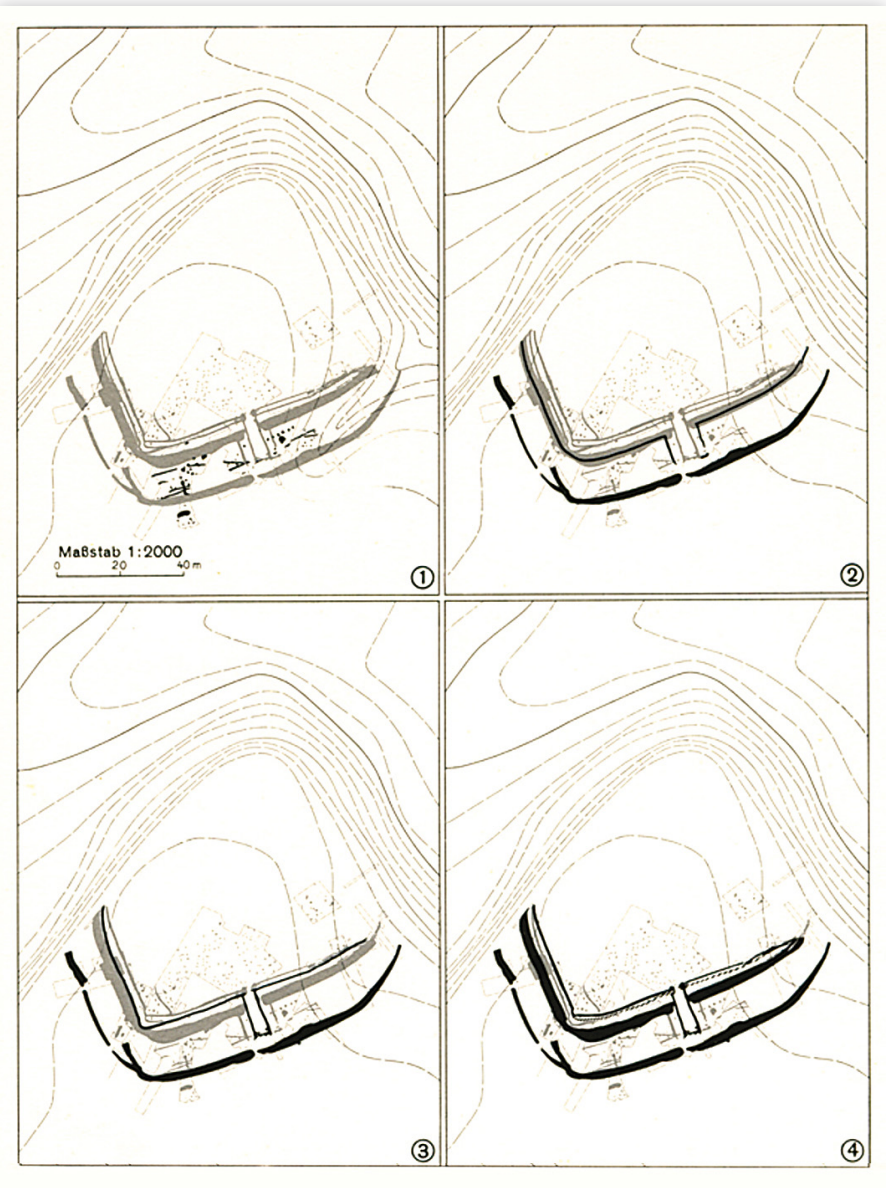

Malgré ses particularités, en effet, ce site a été rapproché, à juste titre, de la catégorie des Herrenhöfe (fermes seigneuriales) qui sont fréquentes, à la fin du Premier âge du Fer, dans la même zone géographique de l'Allemagne sud-orientale, et souvent encloses dans des systèmes de fossés et palissades multiples et sophistiqués [13]. Le rôle joué par les délimitations complexes de ces établissements a été diversement interprété, mais on tend aujourd'hui à en minorer la fonction défensive.

Ainsi, c'est plutôt à une analyse sociologique que semble se prêter le cas de l'établissement enclos de EnkeringGatzäcker, à Kinding (Allemagne, Bavière, Lkr. Eichstätt). On peut suivre, en effet, l'évolution de l'occupation dans ce secteur depuis le Bronze final, époque où se côtoient plusieurs noyaux d'habitats ouverts, exempts de tout système de délimitation. Au Hallstatt ancien, un établissement enclos, dont plusieurs phases ont été reconnues, se juxtapose à un « hameau » ouvert qui se maintient vers le sud et l'est. Pour le dernier état de l'enclos, qui semble comporter quatre lignes de clôture imbriquées les unes dans les autres (fig. 12), on peut reconstituer, en fait, deux phases de construction : à la plus ancienne appartiennent le fossé et la palissade extérieurs (avec les bâtiments 16,18 et 23 ) ; à la plus récente le fossé et la palissade intérieurs. Dans ce tout dernier état, la palissade interne est doublée sur ses quatre côtés par des alignements de trous de poteau, ce qui rappelle l'architecture rencontrée au Britzgyberg (supra). C'est d'ailleurs la proposition de la présence d'une sorte de « chemin de ronde » qui est retenue par Sabine Kas dans sa publication monographique du site [14] et elle appuie cette interprétation en particulier sur la profondeur (relative) des trous de fondation (40 à $50 \mathrm{~cm}$ ) qui permettrait d'atteindre une certaine hauteur de construction. L'hypothèse est complétée par celle d'une série de cases servant de «magasins de stockage » sous le chemin de ronde. Pourtant la situation de ce quadrilatère interne, la distance qui le sépare du fossé avec lequel il a dû fonctionner (fossé interne), et dont l'interruption côté sud ne semble en aucune manière fortifiée, ni même protégée, la présence au sein de l'enclos interne d'un unique bâtiment en position centrée, tout ceci paraît dénoter plutôt la recherche d'une certaine monumentalité, voire celle d'une architecture « de prestige ».

Dans ses travaux récents sur cette région de la Franconie méridionale, Markus Schußmann [15] a d'ailleurs privilégié, pour expliquer l'évolution de l'occupation de ce

[13] Pour la définition et les caractéristiques de ces établissements enclos : Reichenberger 1994 ; LeIdorf 1996.

[14] KaS 2006.

[15] Schussmann 2010. 


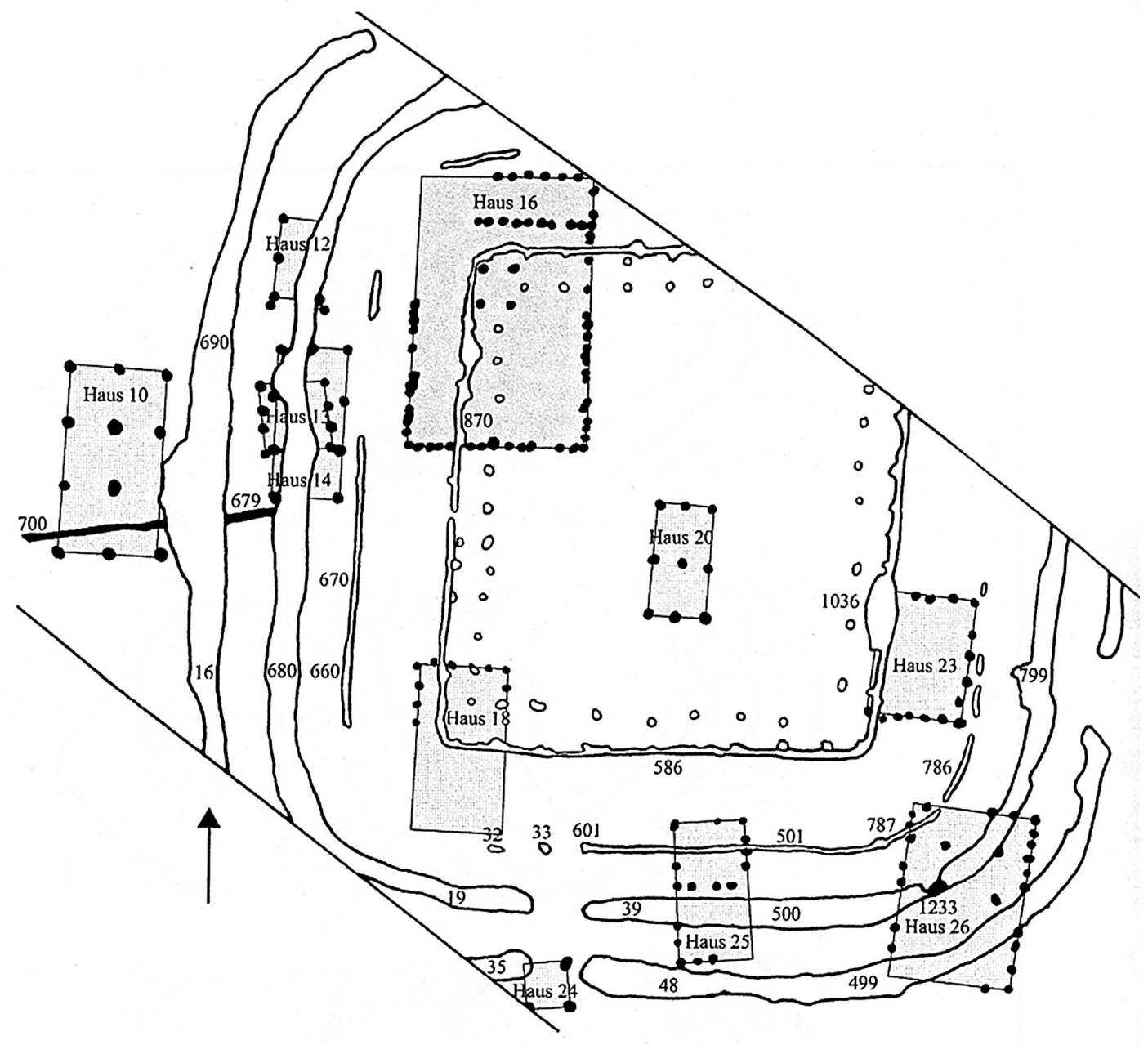

Figure 12

Plan général du Herrenhof de

Enkering-Gatzäcker, derniers états de l'enclos (d'après KAS 2006, Fig. 17, p. 90). secteur depuis le Bronze final, une interprétation en termes socio-économiques, avec l'idée de l'émergence, au début du Hallstatt, d'un groupe dominant qui « matérialise » sa position en isolant son domaine du reste de I'habitat. La ferme seigneuriale ainsi constituée peut logiquement avoir évolué elle-même vers une monumentalité accrue dans la dernière phase de son existence.

\section{CONCLUSION}

Si le désir de protéger les aménagements internes à la grande palissade du Britzgyberg n'a pas été totalement étranger à la construction de celle-ci, et peut expliquer en partie les proportions imposantes de ce dispositif, il ne faut donc sans doute pas surestimer les intentions défensives. Les différents cas analysés ci-dessus semblent confirmer plutôt que, au-delà du souci de structurer l'espace, en marquant peut-être des limites de propriété, le principal objectif de ces constructions a été de rendre visible la démarcation entre différentes catégories sociales, de matérialiser le prestige du chef, en l'isolant du reste de la communauté, ou au contraire, en regroupant autour de lui tous ses dépendants, au sein d'une résidence enclose de façon ostentatoire. N'oublions pas toutefois que cette fonction symbolique concerne en fait tous les types d'enceintes, même les plus puissamment fortifiées, qui, outre leur utilité pratique et défensive, marquent aussi, de diverses manières selon les époques et les cultures, la séparation entre deux mondes. 
Adam, Anne-Marie, 2010a, «Genese und Entwicklung der befestigten Höhensiedlung auf dem Britzgyberg in Illfurth (HautRhin, Frankreich) », dans Dirk Krausse (éd.), « Fürstensitze » und Zentralorte der frühen Kelten, Abschlusskolloquium des DFGSchwerpunktprogramms 1171 in Stuttgart, 12.-15.Oktober 2009, Stuttgart, p. 365-375.

Adam, Anne-Marie, 2010b, « Les systèmes fortifiés du Britzgyberg à Illfurth (Haut-Rhin) : pour une relecture des données », dans Stephan Fichtl (éd.), Murus celticus. Architecture et fonction des remparts de l'âge du Fer, Table ronde internationale de Bibracte 11-12 octobre 2006, Glux-en-Glenne (Collection Bibracte, 19), p. 37-43.

Bérenger, Daniel, 1997, Die Wallburg Babilonie, Stadt Lübbecke, Kreis Minden-Lübbecke, Münster (Frühe Burgen in Westfalen, 12). Bérenger, Daniel, 1999, «Älter eisenzeitliche Burgen in Westfalen ? », dans Albrecht Jockenhövel (éd.), Ä/tereisenzeitliches Befestigungswesen zwischen Maas/Mosel und Elbe, Internationales Kolloquiumam 8. November 1997 in Münster anläßlich des hundertjährigen Bestehens des Altertumskommission für Westfalen, Münster, Aschendorff, p. 99-110.

Bofinger, Jörg \& Goldner-Bofinger, Anita, 2008, « Terrassen und Gräben - Siedlungsstrukturen und Befestigungssysteme der Heuneburg-Vorburg », dans Dirk Krausse (éd.), Frühe Zentralisierungs- und Urbanisierungsprozesse. Zur Genese und Entwicklung frühkeltischer Fürstensitze und ihres territorialen Umlandes, Kolloquium des DFG-Schwerpunktprogramms 1171 in Blaubeuren, 9.-11. Oktober 2006, Stuttgart, p. 209-227.

Diemer, Georg, 1991, Der Bullenheimer Berg und seine Stellungim Siedlungsgefüge der Urnenfelderkultur Mainfrankens, Kallmünz (Materialhefte Bayerischer Vorgeschichte, A 70).

KAS, Sabine, 2006, Enkering-Gatzäcker. Eine Siedlung der späten Urnenfelder- und der Hallstattzeit mit Grabenanlage, Büchenbach.

KuRz, Siegfried, 2010, «Zur Genese und Entwicklung der Heuneburg in der späten Hallstattzeit », dans Dirk Krausse (éd.), «Fürstensitze » und Zentralorte der frühen Kelten, Abschlusskolloquium des DFG-Schwerpunktprogramms 1171 in Stuttgart, 12.-15. Oktober 2009, Stuttgart, p. 239-256.

LeIDORF, Klaus, 1996, Sievers \& Martin Schönfelder (éd.), Die Frage der Protourbanisation in der Eisenzeit, Akten des 34. International Kolloquiums der AFEAF, Aschaffenburg, mai 2010, Bonn, p. 91-113.

Parzinger, Hermann, 1998, Der Goldberg. Die Metallzeitliche Besiedlung, Mainz (Römisch-Germanische Forschungen, 57).

Pätzold, Johannes \& SchwaRz, Klaus, 1961, « Einspät Hallstattzeitlicher Herrensitz am Kyberg bei Oberhaching im Landkreis München: kurzer Vorbericht zu den Ausgrabungen 1959 bis 1961 », Jahresbericht der Bayerischen Bodendenkmalpflege 2, p. 5-15. Reichenberger, Alfred, 1994, «"Herrenhöfe" der Urnenfelder- und Hallstattzeit », dans Archäologische Untersuchungen zum Übergang von der Bronze - zur Eisenzeitzwischen Nordsee und Kaukasus, Regensburg, p. 187-215.

SchaUeR, Peter, 2000-2001, « Umwehrte Höfe in Höhenbefestigungen der Urnenfelderzeit Bayerns », Jahresbericht der Bayerischen Bodendenkmalpflege 41-42, p. 73-84.

SchussmanN, Markus, 2010, « Siedlungshierarchien und Zentralisierungsprozesse in der südlichen Frankenalb zwischen dem 9. Und 4. Jh. v. Chr. », dans Dirk Krausse (éd.), « Fürstensitze » und Zentralorte der frühen Kelten, Abschlusskolloquium des DFGSchwerpunktprogramms 1171 in Stuttgart, 12.-15.Oktober 2009, Stuttgart, p. 129-167.

SchussmanN, Markus, 2012, Siedlungshierarchien und Zentralisierungsprozesse in der Südlichen Frankenalb zwischendem 9. und 4. Jh. v. Chr., Rahden/Westf. (Berliner Archäologische Forschungen, 11).

Schwertzer, Joël, 1997, « L'oppidum du Britzgyberg et le facies hallstattien dans le Horst de Mulhouse », dans Patrice Brun \& Bruno Chaume (éd.), Vix et les éphémères principautés celtiques. Les VI ${ }^{e}-V^{e}$ siècles avant J.-C. en Europe centre-occidentale, Actes du colloque de Châtillon-sur-Seine, 27-29 octobre 1993, Paris, p. 57-66. 